\title{
The Rise and Fall of Napster - an Evolutionary Approach
}

\author{
Bengt Carlsson, Rune Gustavsson \\ Blekinge Institute of Technology,371 25 Ronneby, Sweden \\ E-mail: bengt.carlsson@bth.se, rune.gustavsson@bth.se
}

\begin{abstract}
The paper addresses dynamics in information ecosystems due to competition between selfish agents to get control of protectable resources. In our case study we investigate the first arms race on Internet triggered by the Napster introduction of an easy to use service for sharing files with music content among users. We set up a model for investigation of possible scenarios emerging from the Napster and Gnutella peer-to-peer tools for information sharing. We also introduce a formal model for analyzing the Napster scenario in the cases of selfish or altruistic users. The prediction provided by our model is in line with what really happened in the Napster case. The model also shows that the outcome was indeed unavoidable if we have selfish users.
\end{abstract}

\section{Introducing Napster and Gnutella. Setting the Scene}

The file format MP3 introduced the possibility to compress large audio files into more easy to handle files. For music lovers it became possible to store lots of MP3 files on computers or share them with others, using an Internet connection and file transfer protocols. Napster introduced a peer-to-peer (P2P) environment providing easy mechanisms for connecting different users (peers) and their MP3 file resources. Each of the peers (users) could use a lookup service provided by Napster to find out where to retrieve wanted MP3 files, and also provide their own files. That is, Napster provided a centralized register of locations of files but the files themselves remained at the individual users. Napster quickly became a tremendous success with almost a hundred million of users downloading MP3 music for free. Of course Napster simultaneously become a threat against traditional business models supported by the record companies.

The fight initiated by Napster and taken up by Recording Industry Association of America (RIAA) was about ownership of content and enforcement of copyright laws. At the start Napster concentrated its efforts on providing easy MP3 file sharing among users willing to share their files with others. So, in a sense Napster itself did not 'copy' any files, it only provided easy means for others to share content between each other. After some heated debate about 'freedom on the net' and 'rights to content' there were in the beginning of March 2001 a court order forcing Napster to protect copyright, of music from being shared using its centralized lookup service. The mechanism for protection was to introduce filtering of the lookup service. Users, groups of users or others who of different reasons wanted to protect 'freedom on the Net' quickly circumvented the first simple filters. In short the first information war on 
the web become a reality, including an arms race concerning the control of distributed and valuable content.

The following figure captures the principal players and issues of the battlefield.

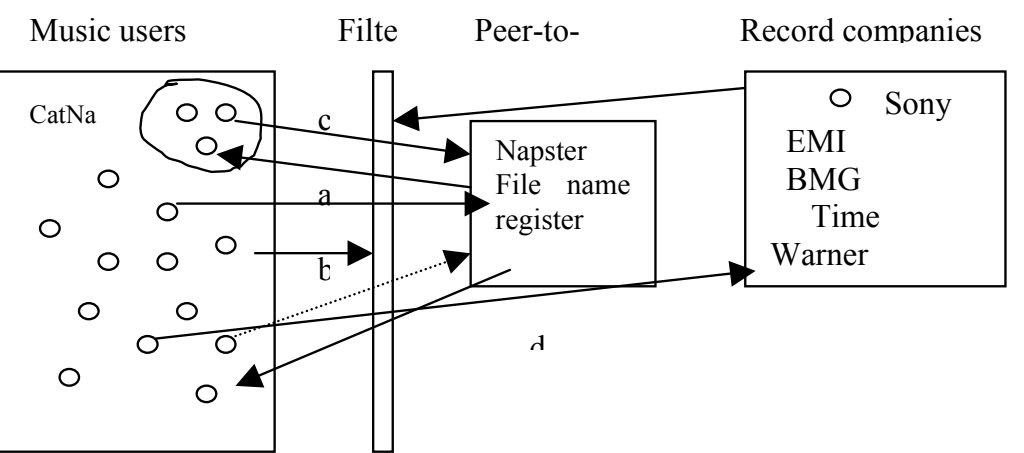

Figure 1 Information warfare in a Napster clone

CatNap is a local proxy server that uses letter shifting (cryptography) of all data sent to the Napster server. The filters on Napster's servers no longer recognize the filenames. The drawback is that users only find files of other CatNap users on the Napster network.

In Figure 1 we have indicated participants of the 'content war' as users, arms providers, e.g., CatNap, and record companies which all form alliances and create new weapons to win the war. In this paper we will model the situation as an information ecosystem of competing agents or groups of agents. The dynamics of the 'content war' is interesting to model for several reasons. Firstly, we know at the time of the publishing of the paper, the outcome. Napster has, at least temporarily, closed down. Could there have been other outcomes? Secondly, what can we learn from this example in order to design information ecosystems with desirable properties.
User - distributed peer-to-peer
Record companies

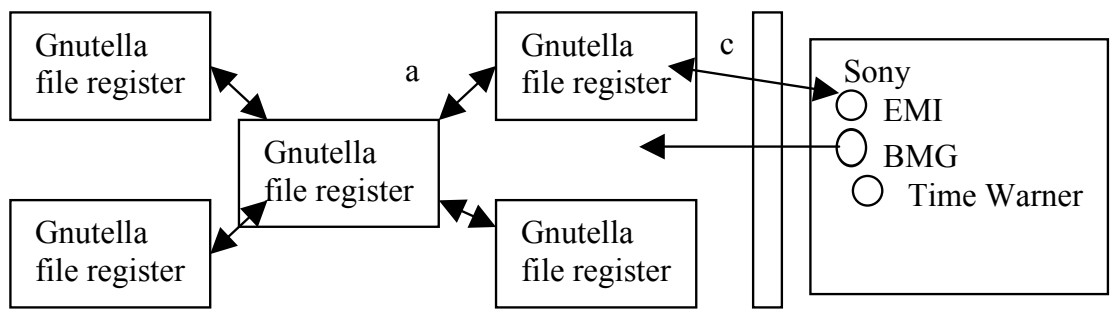

Figure 2 Information warfare in a Gnutella clone

During the war other P2P providers for content sharing appeared. The best example is Gnutella. Gnutella does not have a central file register as Napster. The 
RIAA weapon against Napster, enforcing a filter at the Napster site was not applicable in the Gnutella case. Figure 2 captures the battlefield at Gnutella.

The Gnutella based 'content war' is different due to the distribution of the lookup services. We will compare these two P2P systems, e.g., what would have happened if Napster had had a distributed lookup service? What would have been a proper first RIAA counterattack?

The rest of the paper is organized as follows. In the next section we discuss the characteristics of the two types of warfare described above. The following section gives a short description of the actors in the warfare. The warfare is modeled as a competition between selfish agents. We have elsewhere addressed other issues of this model (Carlsson 2001). A formal model for analyzing and prediction a Napster type of 'content war' is proposed. We conclude the paper with a summary and some open questions.

\section{Discussion of the Napster and Gnutella Warfare}

Napster and Gnutella exemplifies a centralized and a distributed method for information exchange. Both systems have been involved in an arms race within information warfare. In figure 1 the centralized Napster clones are examined and in figure 2 the distributed Gnutella clones. Both systems presuppose societies of selfish actors, but the success of an agent as part of an antagonistic group is dependent on the degree of cooperation within the group.

The "main battle" in figure 1 is about the filtering function. There are several possible scenarios following the introduction of the filtering function.

1. The users copyrighted files are filtered out, making Napster a music distributor respecting copyright laws. If users continue using Napster and if the filtering function is working properly the record companies will be the winners against the users. If users are leaving Napster for other P2P tools, such as Gnutella, Napster will be the loser. Record companies and users will in that case continue an arms race without Napster's de facto standard of $\mathrm{P} 2 \mathrm{P}$ tools.

2. The users try to dupe the filtering function in a conscious or unconscious way. This alternative presumes a cooperative behavior among users, because a user will benefit from the activity of others. A too widespread altering of file names will probably make the filtering function more alert. The arms race concerning the filtering function will probably reach a dead end. The record companies have a leaky filter and the users have an incomplete MP3 record register. Both sides may find better alternatives.

3. A subgroup of users encrypts their MP3 files. CatNap dupes the filter and uses the Napster serves as if no filter existed. This will be a victory for the users, if the Napster server remains unaffected. With an analogy to biology, CatNap will live as a parasite. If a parasite kills its host, the breeding-ground will disappear. Why should Napster keep its server if all users join the CatNap? In reality, CatNap disappeared during the first month of a filtered Napster community.

4. The users join the record companies music sites to pay for downloaded copyrighted music. Record companies have the opportunity to offer additional 
(non digital) materials, concert schedules for the artists or introducing free downloadable music from new artists (lowering their commercial introduction costs).

Due to our formal analysis of section 3, we do not think other centralized P2Ps will take over Napster's position; neither will encryption tools be more dominating. RIAA will sue imitations of Napster and the filtering function should limit the spread of encrypted files for the great majority of people. A sub-community of music sites with unfiltered files will keep the arms race alive.

Since Napster's filters was introduced half a year ago, other unfiltered MP3 tools have grown enormously- MusicCity, a hybrid between Napster and Gnutella, has grown more than forty-fold now reaching more than one million users a day. During one week more than 1.500 .000 copies of MusicCity were downloaded compared to less than 10.000 for Napster.

Figure 2 shows how a single user is connected to, at most, several thousand other users within a distributed P2P tool. There will be an indirect battle between the users and record companies in the Gnutella case because of the lack of centralized P2P servers. There are at least three perceivable battle zones.

1. Distributed P2P means heavily increased network loads. This "bandwidth stealing" may cause an arms race against net traffic suppliers. In the future more efficient search methods or methods for scaling up the number of nodes may be expected. The search improvement may use agent technology and the scaling up improvement may use a client/server system where clients are hidden behind reflectors.

2. Being part of a file sharing system implies a lot of trust towards other users. In fact virus attacks have happened among Gnutella users. An arms race is supposed to occur where it is in the interest of the record companies to be the bad guys supporting the intruders.

3. Anyone may join the open P2P society, not just ordinary users. It is in the interest of record companies and other commercial companies to introduce improved $\mathrm{P} 2 \mathrm{P}$ tools with spyware facilities included. These tools log the users' habits and may use the information for commercial or security interests. An arms race is already ongoing with anti-spywares detecting the spywares.

If Napster and RIAA block downloading, a distributed P2P file transfer is favored. We do not think any of these tools may fully replace Napster because of the increased net loads. Instead of being sued by RIAA, the net traffic suppliers may react against a too successful distributed P2P system.

A robust Information Ecosystem may evolve as a result of arms race situations as in the Napster/Gnutella cases. The advantage of participating in an arms race is not a permanent but a time-extended robustness; to be able to download an MP3 file before the filtering agent observes it or, to avoid a virus attack because of a new anti-virus tool being available. Agents who fail will be culled or have to improve their robustness in relation to mainstream agents. Within Information Ecosystems those parts under attack may improve, making future attacks less successful due to arms race.

Viruses and spywares are tools that may infiltrate or attack the Information Ecosystem. Firewalls against virus attacks (and maybe against spywares in the future) will increase the protection. Anti-virus and anti-spywares increase the ability for the 
intruders to be detected and the response of being weakened. An arms race will occur when countermeasures are taken by the intruding part.

\section{Actors in the Content Wars}

We will in this section describe three actors within Information Ecosystem. Two of them, the record companies and the music listeners are each forming an antagonistic part of the ecosystem. The P2P providers may form coalitions with both the record companies and the music listening users from time to time.

The main goal for the record companies is to have users pay for downloading copyright protected music. If users download for free they will probably not buy records. This seems as an obvious position taken by the record companies, but there are objections for the record companies to defeat Napster as a source of illegal distributor:

- Napster makes it possible for the record companies to reach millions of presumptive customers for introducing new artists or promote new releases.

- Napster is a (at least partly) loyal site towards the record companies, which is shown by the alliance with Bertelmann AG, one of the big record companies.

- It is probably impossible to lock out all copyrighted music from the Napster servers, because of insufficient technical solutions and improved efforts from users to outwit the server.

- If Napster shuts down or becomes a pay-for site, a lot of other P2P sites with a more hostile attitude against the record companies may take over.

If consumers are not willing to pay for downloading music, they will still have a lot of opportunities with or without Napster. Some record companies and artists realize a risk of losing control if Napster disappears, while other regard Napster as the root of all evil. This dynamic determines the actions of the recording industry.

The main interest from the users' point of view is to download free music as simply as possible. Unlike the big economic interests from RIAA, there is no essential question binding users together. So why should users cooperate with other users?

Within sociobiology one kind of cooperative behavior is explained as "if you scratch my back, I scratch yours", or expressed differently; I can do you a favor if it does not cost too much and if I can expect something in return. Within the Napster domain we ask whether it is possible for distributed rules to appear within file sharing systems, without any obvious advantages for the user giving this service. As a group the users should keep together but a single user should try to keep the advantage of file sharing without giving services back, if there is a risk involved.

Users should be aware of the risk of connecting to other users, because they are actually sharing file systems located inside a user's computer. There is a risk of having a virus attack or being exploited by spywares..

Napster initiated a P2P information exchange revolution at the same level as Netscape and later on Microsoft Explorer browsers did within Internet. Unlike the major Internet browser tools, it is not sure Napster will put an industrial standard to the P2P area. Napster is not holding a monopoly over a specific computer service; it has rival clones and options, which may quickly take over if things go wrong. With 
P2P tools, the files stay on the client machine, never passing through the server. Napster clones provides the ability to search for particular file names on one or more servers before initiating a direct transfer between the clients. Gnutella clones have a real P2P connection and do not store any information at central servers.

\section{A Formal Framework of Content War}

We restrict our discussion to 'content war' of the Napster type. We model the arms race as a discrete set of events. At time $t_{n}, M_{n}$ denotes the number of copyright protected files at Napster, $N_{n}$ denotes the number of files protected by the filter at the same time. The protection rate, $P_{n}$, at the same time is given by:

$$
p_{n}=\frac{N_{n}}{M_{n}}
$$

where $P_{n}<1$, and the users can download $U_{n}=M_{n}-N_{n}$ files.

From (1) it follows that we have total protection of the copyrighted material if and only if $\lim _{n \rightarrow \infty} p_{n}=1$

The arms race is modeled as two potentially infinite sequences. The defense sequence $D=\left\{M_{1}, M_{2}, \ldots,\right\}$ and the attack sequence $A=\left\{U_{1}, U_{2}, \ldots,\right\}$ The arms race will continue only if it is worthwhile to use the lookup service at Napster to download files during any given protection rate. At any time $t_{n}$ the attackers will develop new tools to penetrate the filter of the $t_{n}$ generation protecting $M_{n}$ that will enable users to access extra files during the time interval up to time $t_{n+1}$, when the arms race have produced the next generation of filter mechanisms.

The incentive for users to use the lookup service at Napster in the time interval $\left[t_{n}, t_{n+1}\right]$ is:

$$
I_{n}=\left(U_{n}+q_{n} M_{n}\right) / M_{n},
$$

where $0<q_{n}<1$, and $q_{n} M_{n}$ is a measure of the gain the users will have due to better attack tools between the generation updates of the protective defense.

A continuous arms race presupposes that the incentive for selfish users to visit Napster is always positive during the arms race, i.e.,

$$
\lim _{n \rightarrow \infty}=a>0
$$

From (1) and (3) we derive $\lim _{n \rightarrow \infty}=a$, and we have the asymptotic behavior of the downloaded files by an eager and selfish agent 
$U_{n+1}=U_{l}+\sum_{k=1}^{n} q_{k} M_{k}$

From this asymptotic behavior we can deduce the following inequality:

$n \leq K\left(M-U_{l}\right) / a M_{l}$,

where $K$ is a constant and $M$ is the maximum number of copyrighted files.

From this upper bound estimate of $\mathrm{n}$ we thus conclude that the arms race eventually will cease due to lack of incentive to continue using the services for the users even if total protection of copyrighted material has not been achieved, i.e, we can not have (3). We also can conclude that in the case of unselfish agents, e.g., $a=0$ in (3), $n$ can be unbounded and hence we can an indefinite arms race. This means that users will continue to use the services even if there are no personal gains after a while. The reason could be altruistic, e.g., to contribute to the development of total copyright protection on the web.

We thus can predict that a Napster type of 'content war' eventually will end with a loss for Napster in the sense that selfish users will lose interest in the Napster services! Compare with the scenarios of Section 2. This prediction is in line what really happened to Napster.

\section{Conclusions}

One of the most interesting events, which have occurred during the last year on the web, is the Napster-Gnutella introduction of peer-to-peer based information sharing tools. The response from the music industry started the first 'content war' on the net triggered by a court law forcing Napster to install filtering protections for copyrighted music files. The countermeasures, i.e., tools to circumvent the installed filters quickly appeared followed by improved filtering mechanisms. We have in fact witnessed an arms race on the Internet.

The Napster content war will undoubtedly be followed by other similar conflicts and emergent behaviors in Information Ecosystem. By now, we know the outcome of the war, Napster has (temporary) lost. We introduce in the paper a formal model of arms race in Information Ecosystems in order to analyze the Napster case and to allow more general conclusions.

We use an evolutionary model based on natural selection, i.e. measuring the "fitness" of the individual agents to explain long-term adaptations, e.g., hardening of protection mechanisms, in the Information Ecosystem.

We regard the hardening of protection mechanisms of assets to be the result of an arms race where protection measures are followed by sharper weapons of attack in an escalation model. In order to model an ongoing escalation we introduce selfish and eager attacking agents and eager defending agents.

Our theoretical model shows that during these assumptions the arms race will end with a loss for the attacking side, as in the Napster case. If the attacking agent is not selfish but only eager, we can have an infinite arms race. Our model is in fact more 
general than the Napster case. A Gnutella type of 'content war' could be modeled in the same way.

In reality a Napster war ends earlier than the model predicts simple due to the fact that there appear other options, which means that a selfish agent might decide to leave Napster for that reason. This is in fact what has happened on the web.

One should observe that in the 'content war' mentioned the only service, which could be effectively blocked by the music industry, was the centralized Napster service. We could foresee that in future Information Ecosystems more and more of the services will be 'hardened' to protect 'content ownership' and thus enable 'download' by payment only. What our formal model predict in these cases, since the 'free alternatives' will be scare or non-existent, is that selfish agents eventually will pay for their downloads than continue the arms race for free down loading of copyrighted material. That is, we will have a situation with an emerging worldwide price war on content on the net.

An interesting future work is to further elaborate these issues by extending the model to open Information Ecosystems.

We furthermore believe that an approach using methods of investigation similar to a biological ecosystem will be necessary in the future of the Information Ecosystem.

Acknowledgements: Besides the anonymous reviewers we also want to thank Martin Hylerstedt for proof-reading.

\section{References}

Carlsson, B. The Tragedy of the Commons - Arms Race within Peer-to-Peer Tools. To appear in the proceedings of the 2nd International Workshop Engineering Societies in the Agents World. ed. Omicini, A., Petta, P., and Tolksdorf, R. Lecture Notes in Artificial Intelligence 2203, Springer-Verlag (2001)

Carlsson, B. and Davidsson, P. A Biological View on Information Ecosystems. To appear in the proceedings of the Second Conference on Intelligent Agent Technology, World Scientific (2001)

Carlsson, B. and Gustavsson, R. Arms Race Within Information Ecosystems. To appear in the proceedings of the Fifth International Workshop Cooperative Information Agents, SpringerVerlag. (2001)

Dunbar, R., Grooming, Gossip and the Evolution of Language, Faber \& Faber Ltd (1996)

Sandholm,T.W., and Lesser, V.R., Coalitions among computationally bounded agents, Artificial Intelligence, 94(1) (1997) 99-137

Wellman, M., A computational market model for distributed configuration design, Proc. 12th National Conference on Artificial Intelligence (AAAI-94), Seattle, WA (1994) 401-407

Williams, G. C., Adaptation and natural selection, Princeton University Press (1966)

Wilson, E. O., Sociobiology-The abridged edition, Belknap Press (1980) 